\title{
Auxiliary Variables for Deformable models
}

\author{
Laurent D. COHEN \\ CEREMADE, Université Paris-Dauphine, 75775 Paris 16, France \\ cohen@ceremade.dauphinc.fr
}

\begin{abstract}
We present a new mathematical formulation for curve and surface reconstruction algorithms by introduction of auxiliary variables. For deformable models and templates, two-step iterative algorithms have been often used where, at each iteration, the model is first locally deformed according to the potential data attraction and then globally smoothed.

We show how these approaches can be interpreted as the introduction of auxiliary variables and the minimization of a two-variables energy. This permits to transform an implicit data constraint defined by a non convex potential into an explicit convex reconstruction problem.

We show some mathematical properties and results on this new auxiliary problem, in particular when the potential is a function of the distance to the closest fea. ture point. We then illustrate our approach for some deformable models and templates and image restoration.

Keywords: Deformable Models and Templates, Distance Map, Energy Minimization, Image Restoration, Pattern Matching, Spline Functions, Surface and Curve Reconstruction.
\end{abstract}

\section{Introduction}

Many problems in Computer Vision are formulated by the minimization of an energy. Using deformable models and templates, the extraction of a shape is obtained through the minimization of an energy composed of an internal regularization term and an external attraction potential (data term), examples can be found (full references in [1]) for applications in segmentation, surface reconstruction, image restoration and feature extraction.

The minimization is usually solved by gradient descent using an iterative scheme. Often, to make the task possible or easier, each iteration is divided in two stages which may be interpreted as a separation between a global transform and a local one. It consists in minimizing separately the two terms of the energy. While each step may make one part of the energy decrease, it may globally increase. Our work gives a good mathematical formulation to many of these data extraction and reconstruction algorithms by adding an auxiliary variable in the energy in a way similar to [2] but in a different context.

The original contribution of this work is twofold. First we introduce auxiliary variables to define a twovariable energy for shape extraction and reconstruc- tion and show some mathematical results on its minimization. This permits at each iteration to transform a problem with implicit data constraints defined through the minimization of a potential into a reconstruction with regularization of explicit data.

We then apply these results to some already existing two-step iterative algorithms for deformable templates and deformable models to show how they can be interpreted as a minimization of a two-variable energy. We give by the way a uniform mathematical formulation of many computer vision problems. In general, the energy can be written:

$$
E(v)=\int R(v)+\int P(v)
$$

where $v$ is the unknown shapc, $R$ is the regularization term and $P$ is the potential. Usually $R$ is the norm of a derivative of $v$. In case the model is parametric, the internal energy is not necessary and the potential alone is minimized $[3,4]$. In [5], we introduced the definition of an attraction potential to already extracted edges. In [6], we give a survey of some reconstruction approaches. In particular, we make a distinction between explicit and implicit attraction and give physical interpretations in terms of zero length springs. By explicit attraction, we mean that there are explicit constraints between known points of the space and the shape. By implicit, we mean that the constraint is defined through an attraction potential. This potential is designed in such a way that the low values of $P$ correspond to points and features of interest although we do not know in advance their location. The more frequent examples of implicit attractions are $P=-\|\nabla I\|^{2}$ used in the "snake" model [7], and $P=f(d)$, a function of the distance $d$ to the closest data point (see [6]).

The energy we introduce with the extra auxiliary variable $w$ has this form:

$E_{a u x}(v, w)=\int R(v)+\frac{1}{2} \int\|v-w\|^{2}+\int P_{1}(w)$

For a given $w$, the minimization of this energy with respect to $v$ is an explicit regularization convex problem. For a given estimate of $v$, the minimization of $E_{a u x}$ with respect to $w$ is a problem that can be made convex even if $P$ and $P_{1}$ are not. We show how this minimization is solved in a straightforward way and that there is a way to choose $P_{1}$ relatively to $P$ to make the $w$ minimization convex and to have:

$$
\inf _{w} E_{a u x}(v, w)=E(v)
$$


The consequence is that this auxiliary problem has the same solution in $v$ as the original initial problem (1):

$$
\arg \inf _{(v, w)} E_{a u x}(v, w)=\arg \inf _{v} E(v)
$$

This proves that the alternate minimization of $E_{a u x}$ with respect to $v$ and $w$ solves the same initial problem with energy $E$.

The use of auxiliary variables permits to transform a non convex problem in a two-step minimization where each step is easy to solve and convex.

\section{Introducing Auxiliary Variables}

We will be interested in the typical form of the auxiliary energy (2) where the influence of the data (defined implicitly by a potential $P_{1}$ ) on the unknown $v$ is indirect in the second term through the use of variable $w$. The introduction of auxiliary variables permits to solve the indetermination of the implicit constraint.

We can see a geometric interpretation of the iterative alternate minimization of $E_{a u x}$ as a deformation of the current shape followed by a regularization. This corresponds to a separation between local deformation and global processing. Recall we are looking for a shape $v$ that fits best the data located at the lower values of the potential $P_{1}$. Given a same initial estimate $v_{0}$ for $v^{0}$ and $w^{0}$, we iteratc a two-stage process: Local deformation. Shape $v^{n}$ being fixed, the minimization of $E_{a u x}$ with respect to $w$ is a problem that is made convex even if $P_{1}$ is not. An auxiliary shape $w$ is deformed to be attracted by small values of $P$, and at the same time has to remain close to $v^{n}$. So $v^{n}$ being given, $w^{n}$ solves a trade-off between localization of features and small deformation from $v^{n}$.

Global processing. Auxiliary shape $w^{n}$ being fixed, the minimizer of $E_{\text {aux }}$ with respect to $v, v^{n+1}$ is a regularized reconstruction of $w^{\mathfrak{n}}$ taken as explicit data. This permits to transform the implicit problem (1) into an explicit classic problem (5).

This geometric interpretation will be more precise in the examples given in Sections 5 to 9 . In the discrete case, the shape is defined by parameters or nodes and the auxiliary variables $w_{i}$ locate the position of auxiliary nodes. An illustration of the two-step approach is given in Fig. 1 where the potential $P$ is a distance to the data set $S$.

\subsection{Two-step Iterative Algorithm}

Minimizing the energy in $w$ is obtained pointwise by $w_{\min }(s)=\psi(v(s))=\operatorname{ArgMin} M(\phi(v(s), M))$ where $\phi(N, M)=\frac{1}{2}\|N-M\|^{2}+P_{1}(M)$. It turns out that when we minimize the global energy $E_{a u x}(v, w)$ with respect to $w$ first and then $v$, the result in $v$ is solution of the energy minimization $E_{Q}$ analogous to the one of Eqn. (1) with a potential $Q(v)=\phi(v, \psi(v))$ :

$$
\begin{aligned}
\inf _{(v, w)} E_{a u x}(v, w) & =\inf _{v}\left(\inf _{w} E_{a u x}(v, w)\right) \\
& \left.=\inf _{v}\left\{\int R(v)+\inf _{w} E_{2}(v, w)\right)\right\} \\
& =\inf _{v}\left\{\int R(v)+\int Q(v(s))\right\} \\
& =\inf _{v}\left\{E_{Q}(v)\right\}
\end{aligned}
$$

So by solving the auxiliary problem (2), we have found a way to solve the initial problem (1) but with a different potential $Q$ instead of $P$. Let us assume in this section that $Q=P$. We are then able to interpret two-step iterative schemes as the successive minimization of $E_{a u x}$ with respect to its two variables. Let us note:

$$
\begin{aligned}
& E_{1}(v, w)=\int R(v)+\frac{1}{2} \int\|v-w\|^{2} \\
& E_{2}(v, w)=\frac{1}{2} \int\|v-w\|^{2}+\int P_{1}(w)
\end{aligned}
$$

Assume that after $n$ iterations we have the pair $\left(v^{n}, w^{n}\right)$, then the next iteration is:

$$
\left\{\begin{array}{l}
\text { Step 1. Local deformation. Minimizing } E_{2} \\
E_{a u x}\left(v^{n}, w^{n+1}\right)=\inf _{w} E_{a u x}\left(v^{n}, w\right)=E\left(v^{n}\right) \\
w^{n+1}=\psi\left(v^{n}\right) \\
\text { Step 2. Global Smoothing. Minimizing } E_{1} \\
E_{a u x}\left(v^{n+1}, w^{n+1}\right)=\inf _{v} E_{a u x}\left(v, w^{n+1}\right) \\
v^{n+1}=\operatorname{smooth}\left(w^{n+1}\right)
\end{array}\right.
$$

It is obvious that $E_{a u x}$ is always decreasing from one half iteration to the next. From this we can see that $E\left(v^{n}\right)$ is also decreasing.

This works since the two steps can be interpreted as separate minimizations of the same energy $E_{\text {aux } x}$. In the case $P$ and $P_{1}$ do not satisfy the correct hypothesis and this formulation is not possible, there is no warranty for a descent in energy $E$ after a single two-step iteration. Usually, in the minimization algorithms used, each half iteration makes one term of the energy $E$ (the regularization term or the potential term) go down while the second part goes up. In gencral, there is no control on the balance of these two actions and if there is a negative balance, it will not converge. This corresponds to the same oscillations that were mentioned in [5] as instabilities due to image forces. When the potential satisfies the good hypotheses, there is always a descent in the global energy at each half iteration and this ensures convergence of the energy to a minimum.

\section{Resolution using conjugate functions}

Complete results and their proofs can be found in [1]. Let us recall the definition of the Conjugate function (see [8]), also called Legendre transform.

Definition 1 If $\varphi$ is a function from a Euclidean space $\mathcal{E}$ to $\mathbb{R}$, the conjugate function of $\varphi$ is

$$
\varphi^{*}(u)=\sup _{v \in \mathcal{E}}((u, v)-\varphi(v))
$$

We show that

Theorem 1 If $\varphi(N)=\frac{1}{2}\|N\|^{2}-P(N)$ is convex, we have $Q=P$ with $P_{1}$ defined by equality:

$$
\frac{1}{2}\|M\|^{2}+P_{1}(M)=\varphi^{*}(M)=\left\{\frac{1}{2}\|N\|^{2}-P(N)\right\}^{*}
$$


Actually, we now show that since to implement the descent algorithm in (7) only $\psi$ is needed, computation of $P_{1}$ is in fact not necessary once you know it exists and is regular.

Theorem 2 Under the hypotheses of Theorem 1, if $P$ is $C^{2}$ and $\frac{1}{2}\|M\|^{2}-P(M)$ is strictly convex, then the local deformation is $\psi(N)=N-\nabla P(N)$.

This permits to interpret the step of usual gradient descent as a minimization of the auxiliary energy $E_{a u x}$ with respect to the added variable. Since the condition on $P$ is not easy to check for a given potential, we now give a special case of potential for which we have found an expression of $P_{1}$. Moreover, in this case the condition will be weaker and easier to check than that on $P$.

\section{Explicit resolution when $P=f(d)$}

In the case of a potential $P$ defined as a function $f(d)$ of the distance to the closest data point, as in [5], we show that we can find $P_{1}=f_{1}(d)$. 'The results do not depend on the dimension of the space where we consider our objects (2-D or 3-D). Usually we have $f(d)=\alpha \frac{d^{2}}{2}$ or as explained in [6], it can be useful to define $f$ with a returned bell shape like $f(d)=-e^{-d^{2}}$ or a threshold function $f(d)=\inf \left(\alpha \frac{d^{2}}{2}, \beta\right)$. This permits to avoid influence of outlyers by giving the same contribution in the energy to points which are far cnough to the data. When the distance to closest data is large enough, it means that there is no match between this point and data and it is meaningful to consider its contribution to the energy only as a penalty $\beta$ which does not depend on the distance. This means that only the more likely reliable data is used. This is a different formulation for robust statistics. We have shown that in the case $P_{1}(M)=f_{1}\left(d_{S}(M)\right)$, theorem 1 becomes:

Theorem 3 If function $\varphi=\left(\frac{x^{2}}{2}-f(x)\right)$ is convex, defining $f_{1}$ by equality:

$$
\left(\frac{\lambda^{2}}{2}+f_{1}(\lambda)\right)=\varphi^{*}(\lambda)=\left\{\frac{1}{2} x^{2}-f(x)\right\}^{*}(\lambda)
$$

we have $Q(M)=P(M)=f\left(d_{S}(M)\right)$.

This has advantage on Theorem 1 that the computation of the conjugate in Eqn. (9) is now for a function of one real variable instead of two or three. We reduced the problem from any dimension to onc dimension. Also for a given function $f, f_{1}$ does not depend on the data defined by $d_{S}(M)$. It has to be computed only once for all, and a precomputed pair $\left(f, f_{1}\right)$ can be used for all problems. Note also that $f$ may satisfy this condition while $P=f\left(d_{S}\right)$ does not satisfy the condition of Theorem 1 since $d_{S}$ is not convex. This means that the condition here is a lot more general than the previous one. We give an explicit expression of $\psi(N)$ relatively to $f$ which does not need computation of $f_{1}$.
Theorem 4 Under the hypotheses of Theorem 3, if function $\frac{1}{2} x^{2}-f(x)$ is strictly convex and $C^{2}$, then $\psi(N)=N-f^{\prime}\left(d_{S}(N)\right) \nabla d_{S}(N)$.

Example 1 In the simple case where $f(d)=\alpha \frac{d^{2}}{2}, 0<$ $\alpha<1,\left(\frac{d^{2}}{2}-f(d)\right)$ is convex and $f_{1}$ is also quadratic using the formula $\left(\alpha \lambda^{2}\right)^{*}=\frac{d^{2}}{4 \alpha}$ and we have $f_{1}(\lambda)=$ $\frac{\alpha}{1-\alpha} \frac{\lambda^{2}}{2}$.

Example 2 If $f(d)=\operatorname{In} f\left(\beta, \alpha \frac{d^{2}}{2}\right), 0<\alpha<1$, then $\left(\frac{d^{2}}{2}-f(d)\right)$ is convex and we can show that we have a closed form formula for $f_{1}(\lambda)$ :

$$
\left\{\begin{array}{lll}
\frac{\alpha}{1-\alpha} \frac{\lambda^{2}}{2} & \text { if } & 0 \leq \lambda \leq(1-\alpha) \sqrt{\frac{2 \beta}{\alpha}} \\
\beta-\frac{1}{2}\left(\lambda-\sqrt{\frac{2 \beta}{\alpha}}\right)^{2} & \text { if } & (1-\alpha) \sqrt{\frac{2 \beta}{\alpha}} \leq \lambda \leq \sqrt{\frac{2 \beta}{\alpha}} \\
\beta & \text { if } & \lambda>\sqrt{\frac{2 \beta}{\alpha}}
\end{array}\right.
$$

\section{Parametric Deformable Templates}

Deformable Templates can be used with various simple regular shapes defined by a small number of parameters like circles, ellipses and parabola arcs [3], superquadrics and hyperquadrics. Since the shape is imposed and regular, the smoothing term is not necessary. The unknown $\mathcal{A}$ is now a small set of parameters defining the shape $\mathcal{S}_{\mathcal{A}}$ and the energy is of the following form:

$$
E(\mathcal{A})=\sum_{i} P\left(v_{i}(\mathcal{A})\right)
$$

where $\left(v_{i}(\mathcal{A})\right)_{i}$ is a set of nodes which discretize the shape $\mathcal{S}_{\mathcal{A}}$. Beginning with initialization of either a model $\mathcal{A}^{0}$ or a set of points $\mathcal{M}^{0}$, we iterate the following two steps:

Step 1: Model Fitting. A set $\mathcal{M}^{k}=\left(M_{i}^{k}\right)_{1 \leq i \leq n}$ being given in this order, a criteria is minimized to find the set of parameters $\mathcal{A}^{k}$ to define the best fit: $E_{1}^{k}(\mathcal{A})=\sum_{i}\left\|v_{i}(\mathcal{A})-M_{i}^{k}\right\|^{2}$ The minimizing vector of parameters defines $\mathcal{A}^{k}$.

Step 2: Deformation of the set of points. The vector $\mathcal{A}^{k}$ being given, a new set $\mathcal{M}^{k+1}=$ $\left(M_{i}^{k+1}\right)_{1 \leq i \leq n}$ is defined by moving each point $v_{i}\left(\mathcal{A}^{k}\right)$ in the direction of descent of $P$ to minimize $E_{2}(\mathcal{M})=$ $\sum_{i} P\left(M_{i}\right)$ The set of nodes $\mathcal{M}^{k}$ is then replaced for the next step by the new set $\mathcal{M}^{k+1}$ which is usually defined by:

$$
M_{i}^{k+1}=v_{i}\left(\mathcal{A}^{k}\right)-\tau_{i}^{k} \nabla P\left(v_{i}\left(\mathcal{A}^{k}\right)\right)
$$

If $\left(\frac{1}{2}\|N\|^{2}-\tau P(N)\right)$ is convex, or $\left(\frac{1}{2} x^{2}-\tau f(x)\right)$ is convex in the case $P=f\left(d_{S}\right)$, these two steps can be interpreted as the two variables minimization of an auxiliary energy:

$$
E_{\text {aux }}(\mathcal{A}, \mathcal{M})=\frac{1}{2 \tau} \sum_{i}\left\|v_{i}(\mathcal{A})-M_{i}\right\|^{2}+\sum_{i} P_{1}\left(M_{i}\right)
$$


where $\left(\tau P_{1}\right)$ is associated to $(\tau P)$ by equality $(9)$ or (10) of theorems 1 and 3 .

Successive minimization with respect to the two variables $\mathcal{A}$ and $\mathcal{M}$ is as follows: $\mathcal{A}$ minimization corresponds to our first step of model fitting minimizing $E_{1}^{k} ; \mathcal{M}$ minimization corresponds to our second step of deformation.

Remark that the auxiliary variable can be seen as the local deformation of the global model best fit. This is very close to the idea used in deformable superquadrics. So at convergence, $\mathcal{A}$ represents the best fit of the parametric model and $\mathcal{M}$ the local displacement between the model and the data.

\section{Pattern Matching}

The problem of pattern matching can be seen as a special case of deformable templates, where the parameters of the model correspond to the nature of the deformation. Let us see first the case when the match between the model and data is done through an affine transform. We have a curve or surface model defined by a set of points $\mathcal{S}^{1}=\left(X_{i}^{1}\right)_{i}$ and try to match this model to data. The data is a set of points $\mathcal{S}^{2}=\left(X_{j}^{2}\right)_{j}$ and we try to minimize the average distance to data after a rotation or an affine transform $R$ followed by a translation $t$. The energy we are trying to minimize has the form:

$$
E(R, t)=\sum_{i} d\left(R X_{i}^{1}+t, \mathcal{S}^{2}\right)^{2}
$$

where $d\left(R X_{i}^{1}+t, \mathcal{S}^{2}\right)=\inf _{j} d\left(R X_{i}^{1}+t, X_{j}^{2}\right)$. This problem would be very simple if to each point of $\mathcal{S}^{1}$, we knew in advance the point in $\mathcal{S}^{2}$ which corresponds, but this is one of the unknowns of the problem. Therefore, an iterative algorithm composed of two steps is often used to find the minimizing transform (see ICP, Iterative Closest Point algorithm in [9]).

Step 1: Matching. For a previous estimate of the transform $\left(R_{n}, t_{n}\right)$, find the matching between points of the transformed shape $\left(R_{n} \mathcal{S}^{1}+t_{n}\right)$ and $\mathcal{S}^{2}$. This is done finding for each $X_{i}^{1}$ in $\mathcal{S}^{1}$ the closest point $X_{j_{n}(i)}^{2}$ in $\mathcal{S}^{2}$ to $\left(R_{n} X_{i}^{1}+t_{n}\right)$. The output is a list of couples $\left(X_{i}^{1}, X_{j_{n}(i)}^{2}\right)_{i}$.

Step 2: Transform. For a given set of matches, $\left(X_{i}^{1}, X_{j_{n}(i)}^{2}\right)_{i}$, find the best transform $\left(R_{n+1}, t_{n+1}\right)$ $\operatorname{minimizing} \sum_{i}\left\|R_{n+1} X_{i}^{1}+t_{n+1}-X_{j_{n}(i)}^{2}\right\|^{2}$.

The necessity of these two steps comes from the fact that when matching the two sets of points, we do not know in advance which point of the first set corresponds to which point of the second set. The first step makes a tentative match and the second step solve the problem now made easier.

As for deformable templates, we can understand these two steps as the minimization of an auxiliary energy with respect to two variables.

$$
E_{a u_{i i}}((R, t), \mathcal{M})=\frac{1}{2} \sum_{i}\left\|R X_{i}^{1}+t-M_{i}\right\|^{2}+\sum_{i} P_{1}\left(M_{i}\right)
$$

The case of a general non-rigid transform is similar to the snake case dealt in next section.

\section{Active Contour Models}

The snake model introduced in [7] is the minimization of the following energy:

$$
E_{s n}(v)=\int_{\Omega}\left\{w_{1}\left\|v^{\prime}\right\|^{2}+w_{2}\left\|v^{\prime \prime}\right\|^{2}+P(v)\right\}
$$

All we say here is also true in the general case of regularization $R(v)$ like in energy of $(1)$ and also in the case of $3 \mathrm{D}$ deformable surfaces $([6])$.

We show in [5] how the solution of the associated evolution equation

$$
\frac{\partial v}{\partial t}-\left(w_{1} v^{\prime}\right)^{\prime}+\left(w_{2} v^{\prime \prime}\right)^{\prime \prime}=F(v)=-\nabla P(v)
$$

is discretized into a two-step iteration algorithm. The system to solve is

$$
(\mathcal{I}+\tau A) v^{t+1}=\left(v^{t}+\tau F\left(v^{t}\right)\right) .
$$

where $A$ is the stiffness matrix obtained after discretization of the derivative terms either by finite differences or finite elements. It is decomposed in two steps, first a deformation along attraction force, then regularization (see figure 2):

$$
\begin{array}{lll}
\text { Step 1: } & \text { Local deformation. } & v^{t+\frac{1}{2}}=\left(v^{t}+\tau F\left(v^{t}\right)\right) \\
\text { Step 2: } & \text { Regularization. } & v^{t+1}=(\mathcal{I}+\tau A)^{-1} v^{t+\frac{1}{2}}
\end{array}
$$

Equation (15) is called a reaction diffusion equation and our decomposition in two steps could be also seen as a separation between these two aspects: the first stcp represents only reaction while the second represents only diffusion.

Assuming good hypotheses on $P$, these two steps can be interpreted as the alternate minimization of an auxiliary energy $E_{s n}^{a u x}\left(v_{1}, v_{a u x}\right)$ with respect to its two variables:

$$
\int_{\Omega} w_{1}\left\|v_{1}^{\prime}\right\|^{2}+w_{2}\left\|v_{1}^{\prime \prime}\right\|^{2}+\frac{1}{2 \tau}\left\|v_{1}-v_{a u x}\right\|^{2}+P_{1}\left(v_{a u x}\right)
$$

where $v_{1}$ is the feature curve, $v_{a u x}$ is an auxiliary curve and $\left(\tau P_{1}\right)$ is associated to $(\tau P)$ by equality $(9)$ or $(10)$ of theorems 1 or 3 . The $v_{1}$ minimization corresponds to our second step of regularization. The $v_{a u x}$ minimization corresponds to our deformation step.

Notice that in our snake algorithm [5], there is a resampling of the curve from time to time to avoid concentration of nodes in some areas and account for length variation. This may be interpreted as the use of a geometric model of the curve or close to a two step formulation of the shape reconstruction on a varying mesh.

\section{Image Restoration}

We saw in section 7 that the two steps came from the semi-explicit scheme of equation (16). Other algorithms have this same property of being explicit in the non linear part and implicit in the linear part. 
In some restoration problems where there is in the energy $E(u)$ a non linear function of the gradient of the image $u$, each iteration is replaced by two steps. The first minimizes the energy with the previous value of the gradient in this non linear term. The second is a new evaluation of the gradient from the new value of $u$. This may be seen as using an auxiliary linear problem at each time step. We give here two examples:

Anisotropic Diffusion. To solve the evolution equation of anisotropic diffusion ([10]) a kind of twostep algorithm was used by the author. we solve

$$
\frac{\partial u}{\partial t}-\nabla(g(\|\nabla u\|) \nabla u)=0
$$

with usually $g(d)=\frac{1}{\left(1+d^{2}\right)^{m}}$. This is discretized using finite differences:

$$
\begin{cases}\text { Step 1. } & G^{n}=g\left(\left\|\nabla u^{n}\right\|\right) \\ \text { Step 2. } & u^{n+1}-u^{n}-\tau \nabla\left(G^{n} \nabla u^{n+1}\right)=0\end{cases}
$$

Non linear Total Variation. The same kind of equation with $g(\nabla u)=\frac{1}{\|\nabla u\|}$ also appears in a restoration algorithm based on non linear total variation [11] minimizing $\int\left(u_{x}^{2}+u_{y}^{2}\right)^{1 / 2}$. This gives an evolution equation similar to (17) and (18). This could be interpreted as solving an auxiliary problem where the nonlinear term is not time dependent.

Note that in these examples, the original problem is modified by freezing a nonlinear part at each step. This can be also interpreted as smoothing algorithms with variable weights. A first step estimates the weights and a second solves the minimization with these constant weights. Those variable weights algorithms have been transformed using a different duality relation in two variables problems in [12]. This makes use of a function $f_{1}$ such that

$$
\inf _{v} v\|\nabla u\|^{2}+f_{1}(v)=f(\|\nabla u\|)
$$

and defines an auxiliary energy such as:

$$
E_{\text {aux }}(u, v)=\int v\|\nabla u\|^{2}+f_{1}(v)+\int\|u-d\|^{2}
$$

This duality permits to give a closer relation between the anisotropic diffusion and the Blake and Zisserman algorithm. Indeed, the non linear term in the latter energy is:

$$
\int f(\|\nabla u\|)=\int \frac{f(\|\nabla u\|)}{\|\nabla u\|^{2}}\|\nabla u\|^{2}=\int g(\|\nabla u\|)\|\nabla u\|^{2}
$$

and for $f(d)=\operatorname{In} f\left(\beta, \alpha \frac{d^{2}}{2}\right), g(d)=\operatorname{In} f\left(\frac{2 \beta}{d^{2}}, \alpha\right)$. This function is very close to the one used in anisotropic diffusion.

\section{B-Snakes}

We gave in [1] a more precise link between classic snakes and the B-snake model. We show that B-snakes or spline-snakes may be interpreted as the solution of a discretization of a snake energy. This means that the solution may be seen either as the minimum of a data energy among all cubic spline functions or a minimum of a classic snake energy among all functions.

Leitner etal [4] introduced a simplified active contour model they called "Spline-Snakes." This model was also used by many authors with the name of BSnakes. The solution of their snake model is found by deformation of a set of node points submitted to an attraction force and then by curve reconstruction using B-Splines.

Note that in [6], the snake problem is solved using a finite element method. B-Splines can be seen as a special case of finite element, but the main difference is that in the snake-spline model, the energy is only external. The main idea is that since the classical snake energy contains a regularization part and cubic splines already minimize a regularization energy, it may be easier to limit the curve set to cubic spline curves and minimize only the potential energy.

We formulate the method of resolution proposed in [4] as a two-step iterative algorithm beginning with an initial set of data points $\mathcal{M}^{0}$.

Step 1: Spline Fitting. A set of points $\mathcal{M}^{k}=$ $\left(M_{j}^{k}\right)_{1 \leq j \leq m}$ being given, the set of control points $\mathcal{A}^{k}=\left(\alpha_{i}^{k}\right)_{1 \leq i \leq n}$ is determined to minimize the least square error $\left\|\mathcal{M}^{k}-\mathcal{B} \mathcal{A}\right\|^{2}$.

Step 2: External Force. A set of control points $\mathcal{A}^{k}$ being given, the external force is applied separately to each point of $\mathcal{B A}=\left(v_{\mathcal{A}^{k}}\left(t_{j}\right)\right)_{1 \leq j \leq m}$ to define a new set of data points.

Remark that an analogous algorithm was also used for 3-D splines solving the inverse problem of FreeForm Deformations in [13].

These two steps can be understood as the alternate minimization of the auxiliary energy $E_{b s}^{a u x}(v, \mathcal{M})$ :

$$
\int_{0}^{1}\left\|v^{\prime \prime}\right\|^{2}+\sum_{i=1}^{n} \frac{1}{2 \tau}\left\|v\left(s_{i}\right)-M_{i}\right\|^{2}+\sum_{i=1}^{n} P_{1}\left(M_{i}\right)
$$

This formulation gives the link between the original snake like problems and the two-step algorithm minimizing $P$ along all curves in the spline space. This is a further justification for finite element methods[6] or spline-snakes.

\section{Conclusion}

We have presented a new mathematical formulation to shape extraction and reconstruction problems. By introducing auxiliary variables we defined a twovariables energy and showed some mathematical results on its successive minimization with respect to each variable. These variables represent an intermediary reconstructed shape. This permits to transform a problem with implicit data constraints defined through the minimization of a non convex potential into an explicit reconstruction convex problem defined by regularization of known data. We showed a way to make this transformation easily in the case where the 
potential is a function of the distance to the closest data. This kind of potential is more and more used in shape extraction work.

This permits to give a better understanding of many already existing two-step algorithms used for deformable templates and deformable models. This permits also to give a more precise link between snakes and B-snakes. Our work can give a good mathematical formulation to many of these data extraction and reconstruction algorithms to modify current algorithms and ensure that the initial energy is minimized and that the algorithm converges.

\section{References}

[1] Laurent D. Cohen. Auxiliary variables and two-step iterative algoritms in computer vision problems. Technical report 9511, Ceremade, Février 1995.

[2] D. Geman and C. Yang. Nonlinear image recovery with half-quadratic regularization. Technical report, Umass, July 1993. to appear in IEEE Transactions on Image Processing.

[3] A.L. Yuille, P.W. Hallinan, and D.S. Cohen. Feature extraction from faces using deformable templates. International Journal of Computer Vision, 8(3), September 1993.

[4] F. Leitner, I. Marque, S. Lavalléc, and P. Cinquin. Dynamic segmentation: finding the edge with snakesplines. In Proceedings of International Conference on Curves and Surfaces, pages 1-4, Chamonix, France, June 1990. Academic Press.

[5] Laurent D. Cohen. On active contour models and balloons. CVGIP: Image Understanding, 53(2):211218, March 1991.

[6] Laurent D. Cohen and Isaac Cohen. Finite element methods for active contour models and balloons for 2-D and 3-D images. IEEE Transactions on Pattern Analysis and Machine Intelligence, PAMI15(11), November 1993.

[7] M. Kass, A. Witkin, and D. Terzopoulos. Snakes: Active contour models. International Journal of Computer Vision, 1(4):321-331, 1988.

[8] H. Brezis. Analyse Fonctionnelle, Théorie et applications. Masson, Paris, 1983.

[9] J. Feldmar and N. Ayache. Locally affine registration of free-form surfaces. In $I E E E$ Proceedings of Computer Vision and Pattern Recognition 1994 (CVPR'94), Seattle, USA, June 1994.

[10] Pietro Perona and Jitendra Malik. Scale space and edge detection using anisotropic diffusion. In Proc. IEEE Workshop on Computer Vision, pages 16-22, Miami, FL, 1987.

[11] L.I. Rudin, S. Osher, and E. Fatemi. Nonlinear total variation based noise removal algorithms. In Ecoles $C E A$ - EDF - INRIA; Modélisations Mathématiques pour le traitement d'images, pages 149-179, Rocquencourt, France, March 1992.

[12] D. Geman and Reynolds. Constrained restoration and the recovery of discontinuities. IEEE Transactions on Pattern Analysis and Machine Intelligence, 14:367383, March 1992.

[13] Eric Bardinet, Laurent Cohen, and Nicholas Ayache. Fitting 3D data using superquadrics and free-form deformations. In Proceedings of the 12 th IEEE International Conference on Pattern Recognition (ICPR'94), pages A-79-83, Jerusalem, October 1994.

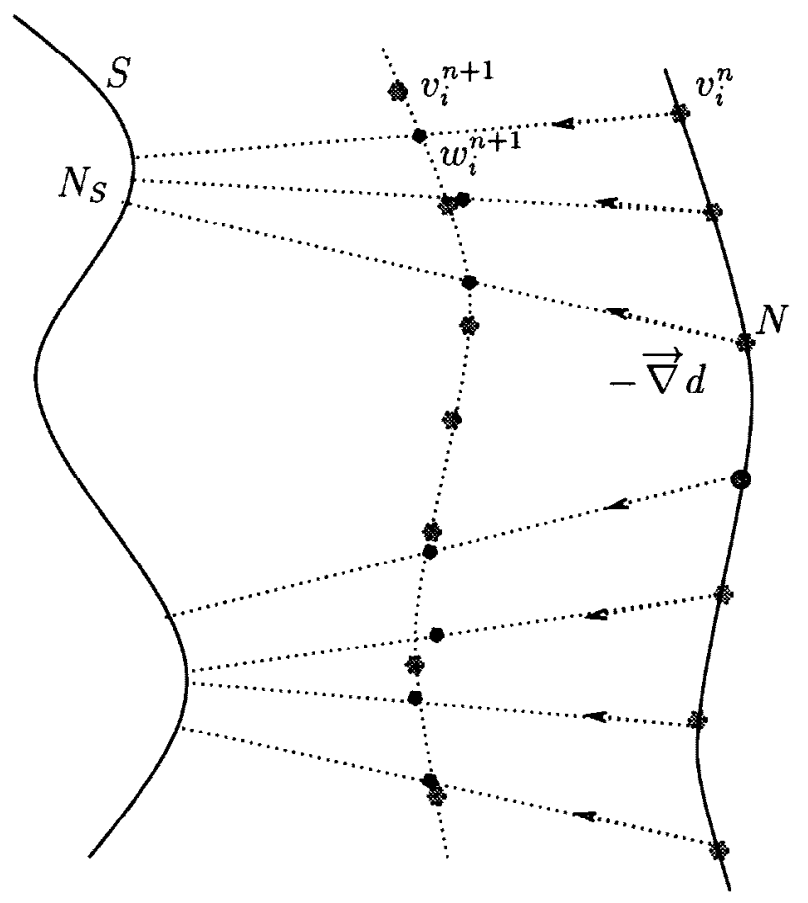

Figure 1: Illustration of one iteration of the $w$ and $v$ minimization. On the left is the data, on the right the current estimation of $v_{i}$ 's and in the middle the minimizing $w_{i}$ 's (black spots). The grey spots and the middle curve represent the new value of $v_{i}$ 's after regularization of the $w_{i}^{\prime}$ s.
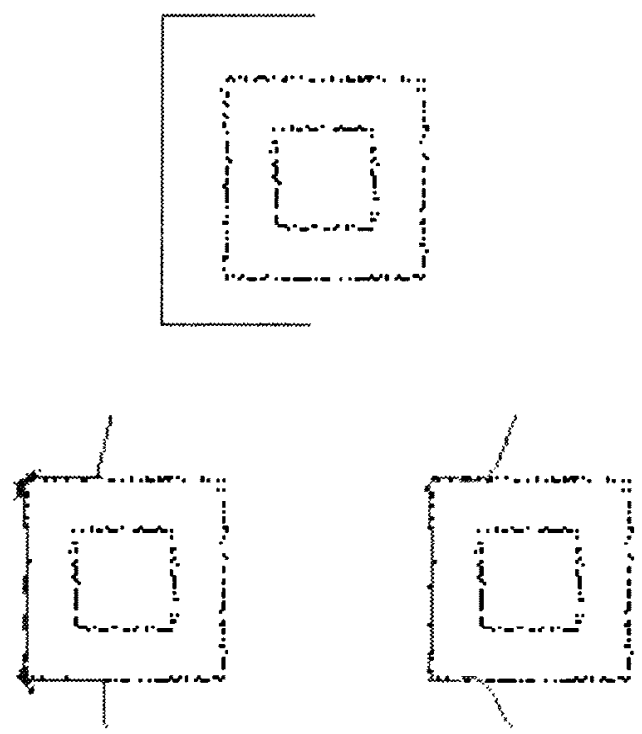

Figure 2: On the top the initial snake followed by the two steps: local deformation and regularization. 